\title{
Knockdown of UCA1 restrains cell proliferation and metastasis of diffuse large B-cell lymphoma by counteracting miR-331-3p expression
}

\author{
MINQING ZHANG ${ }^{1}$, YIPING DU ${ }^{2}$, JINGMEI SHANG ${ }^{3}$, \\ DONGQING ZHANG ${ }^{4}$, XIAOQING DONG ${ }^{5}$ and $\mathrm{HONG} \mathrm{CHEN}^{6}$
}

${ }^{1}$ Department of Clinical Laboratory, Chengwu County People's Hospital, Chengwu Hospital Affiliated to

Shandong First Medical University, Heze, Shandong 274200; ${ }^{2}$ Department of Hematology,

Qingdao Eighth People's Hospital, Qingdao, Shandong 266100; ${ }^{3}$ Department of Imaging,

${ }^{4}$ Department of Public Health and ${ }^{5}$ Emergency Department, The People's Hospital of Zhangqiu Area, Jinan, Shandong 250200; ${ }^{6}$ Department of Clinical Laboratory, People's Hospital of Linyi, Linyi, Shandong 276000, P.R. China

Received June 15, 2020; Accepted October 7, 2020

DOI: $10.3892 / \mathrm{ol} .2020 .12300$

\begin{abstract}
Long non-coding RNA urothelial cancer associated 1 (UCA1) has been reported to act as a carcinogen in bladder cancer, while its role in diffuse large B-cell lymphoma (DLBCL) remains unclear. The present study was designed to explore the expression pattern and role of UCA1 in DLBCL. The expression pattern of UCA1 and microRNA (miR)-331-3p in DLBCL tissues and cell lines were detected by RT-qPCR. Dual luciferase reporter assay was performed to explore the relationship between UCA1 and miR-331-3p. Cell proliferation was explored by MTT assay. Cell migration and invasion abilities were assessed by Transwell assay. In the present study, it was revealed that the expression of UCA1 was significantly upregulated, while miR-331-3p was downregulated in DLBCL tissues and cell lines. Moreover, UCA1 was revealed to competitively bind with miR-331-3p in DLBCL. Functionally, knockdown of UCA1 was revealed to suppress cell proliferation, migration and invasion in DLBCL cells. Furthermore, upregulation of miR-331-3p prevented cell proliferation, migration and invasion in DLBC cells. In conclusion, the present findings firstly demonstrated that UCA1 silencing restrained DLBCL cell proliferation and metastases viability by suppressing miR-331-3p expression. It is suggested that UCA1 could be a possible medicinal target and biomarker for DLBCL.
\end{abstract}

\footnotetext{
Correspondence to: Dr Hong Chen, Department of Clinical Laboratory, People's Hospital of Linyi, 27 Jiefang East Road, Linyi, Shandong 276000, P.R. China

E-mail: puhejia656155@163.com
}

Key words: diffuse large B-cell lymphoma, urothelial cancer associated 1, microRNA-331-3p

\section{Introduction}

Diffuse large B-cell lymphoma (DLBCL) is the most common pathological type of Non-Hodgkin lymphoma (NHL), which accounts for $\sim 25$ to $35 \%$ of NHL in western developed countries and up to $60 \%$ in developing countries (1). The average age of DLBCL onset is 67-70 years. DLBCL is an aggressive B-cell lymphoma that can be cured in $~ 50 \%$ of patients, however $\sim 30 \%$ of DLBCL patients are refractory or eventually relapse (2). DLBCL is the subtype of lymphoma with the worst prognosis (3). Due to the high recurrence and the limitations of current treatment of DLBCL, it is urgent to identify new DLBCL drivers, therapeutic targets and efficacy predictors.

Long non-coding RNAs (lncRNAs) are a type of RNA in eukaryotic cells with a transcript length greater than 200 nucleotides, which are located in the nucleus or cytoplasm, and cannot encode any proteins (4). LncRNAs can bind to proteins, DNA and RNA, and play a wide range of regulatory roles at epigenetic, transcriptional and post-transcriptional levels (5). With the in-depth study of lncRNAs, a growing amount of evidence has revealed that lncRNAs are abnormally expressed in various human tumors and involved in the tumorigenesis and development of human tumors $(6,7)$. Therefore, lncRNAs have been attracting more and more attention as new tumor biomarkers and regulators (8). The role of lncRNAs in the tumorigenesis and development of DLBCL has become a focus of researchers in recent years. LncRNA RP11-513G11.1 was discovered to be highly expressed in DCBCL, and closely associated with the prognosis of DLBCL patients (9). Zhao et al (10) revealed that SNHG14 was upregulated in DLBCL and drove the development and immune evasion by interacting with microRNA (miR)-5590-3p, regulating ZEB1 and PD-L1 checkpoints. Furthermore, SMAD5-AS1 was downregulated in DLBCL, and upregulation of SMAD5-AS1 suppressed cell proliferation by inhibiting miR-135-5p and upregulating APC expression (11).

Urothelial carcinoma-associated 1 (UCA1) was first identified in bladder transitional cell carcinoma, and the 
entire sequence consisted of three exons with a length of $1.4 \mathrm{~kb}(12)$. Scientists have revealed that UCA1 is dysregulated in several human cancers and plays an important role in cancer progression (13). In 2006, upregulation of UCA1 was revealed in bladder cancer, and enhanced cell growth and invasion viability (14). In addition, UCA1 was revealed to be closely associated to tumorigenesis and progression in breast cancer (15), oral squamous cell carcinoma $(16,17)$ and ovarian cancer (18). However, to date, the expression and biological function of UCA1 in DLBCL have not been reported.

The aim of the present study was to determine the expression level of UCA1 in DLBCL tissues and cell lines, as well as the specific functions of UCA1 and miR-331-3p in the progression of DLBCL.

\section{Materials and methods}

Clinical information and cell lines. In total, 38 DLBCL samples (18 males and 20 females; age range: $24-74$ years; mean age: 53 years) and 38 normal samples of adjacent lymph nodes were collected during biopsy from July 2017 to January 2019 at Chengwu People's Hospital (Heze, China). The DLBCL diagnosis was confirmed by histopathological examination, and patients were not treated with radiotherapy or chemotherapy before surgery. The samples were placed in liquid nitrogen for a short time, and then stored at $-80^{\circ} \mathrm{C}$ until use. The inclusion criteria were as follows: i) Patients that were diagnosed with DLBCL according to the WHO classification criteria; ii) patients that had not received radiotherapy or chemotherapy prior to surgery; and iii) patients with complete clinical data and long-term follow-up data. The exclusion criteria were as follows: i) Patients with serious function damage of the heart, liver and kidney; ii) pregnant and lactating women; and iii) previous history of any malignancy. The present study was approved by the Institutional Human Experimentation and Ethics Committee of Chengwu People's Hospital. Moreover, the guidelines of the declaration of Helsinki were strictly adhered to during the study. Prior to the start of the study, all patients signed written informed consent.

In the present study, GM12878 and JeKo-1 cell lines were purchased from the Type Culture of the Chinese Academy of Sciences (Shanghai, China). Other cell lines TMD8, U2932, OCI-Ly-10 and OCI-Ly-7 were obtained from BeNa Culture Collection (Beijing, China). All cells were cultured in a modified Roswell Park Memorial Institute-1640 medium (RPMI-1640), which contained $10 \%$ fetal bovine serum (FBS), $100 \mathrm{~g} / 1$ penicillin and $100 \mathrm{~g} / 1$ streptomycin at $37^{\circ} \mathrm{C}$ with $5 \% \mathrm{CO}_{2}$. Then, $0.25 \%$ trypsin was used to passage the cells every other day.

RNA extraction and reverse transcription-quantitative $(R T-q)$ $P C R$ analysis. Total RNA was isolated from DLBCL tissues and cells according to the manufacturer's instructions using TRIzol reagent (Invitrogen; Thermo Fisher Scientific, Inc.). Then, the total extracted RNA was used as the template for reverse transcriptional cDNA via Transcript cDNA Synthesis kit (Beijing TransGen Co., Ltd.) according to the manufacturer's protocol. The PCR reaction conditions were initial denaturation at $95^{\circ} \mathrm{C}$ for $10 \mathrm{~min}$, followed by 40 cycles of denaturation at $95^{\circ} \mathrm{C}$ for $15 \mathrm{sec}$, annealing at $60^{\circ} \mathrm{C}$ for $30 \mathrm{sec}$, and extension at $72^{\circ} \mathrm{C}$ for $30 \mathrm{sec}$. The primer sequences used were as follows: UCA1 forward, 5'-GCACCCTAGACCCGAAAC TT-3' and reverse, 5'-CCGGACTGCTTCAAGTGTGA-3'; miR-331-3p forward, 5'-GAGCTGAAAGCACTCCCAA-3' and reverse, 5'-CACACTCTTGATGTTCCAGGA-3'; GAPDH forward, 5'-CCTGACCTGCGTGTGGACT-3' and reverse, 5'-GCTGTGGATGGGGAGGTGTC-3'; U6 forward, 5'-CGC TTCGGCAGCACATATAC-3' and reverse, 5'-TTCACGAAT TTGCGTGTCAT-3'. GAPDH was used as the endogenous control of UCA1, while U6 was the endogenous control of miR-331-3p. The expression of UCA1 and miR-331-3p were detected by RT-qPCR with SYBP Premix Ex Taq II (Takara Bio, Inc.) and LightCycler ${ }^{\circledR} 96$ thermocycler (Roche Diagnostics). The $2^{-\Delta \Delta \mathrm{Cq}}$ (19) method was used to calculate the experimental results.

Cell transfection. U2932 cells $\left(4 \times 10^{5}\right)$ in the logarithmic growth phase were seeded into a six-well plate. When cell confluence reached 70-80\%, U2932 cells were transfected with pcDNA3.1-UCA1(UCA1 vector, $40 \mathrm{nM}$ ), small interfering (si)-UCA1 (40 nM), miR-331-3p mimics (40 nM), miR-331-3p inhibitor ( $80 \mathrm{nM})$, or their corresponding negative control (40 nM) by Lipofectamine 2000 (Invitrogen; Thermo Fisher Scientific, Inc.). After transfection at $37^{\circ} \mathrm{C}$ for $1 \mathrm{~h}$, the cells were incubated with complete medium at $37^{\circ} \mathrm{C}, 5 \% \mathrm{CO}_{2}$ and saturated humidity for another $48 \mathrm{~h}$. Vector plasmid, pcDNA3.1-UCA1, miR-331-3p mimics, miR-331-3p inhibitor and the corresponding NC were obtained from Shanghai GenePharma Co., Ltd.. The sequences were as follows: si-UCA1, 5'-GAGCCGAUCAGACAAACAAUU-3'; si-NC, 5'-UUCUCCGAACGUGUCACGUTT-3'; miR-331-3p mimics, 5'-GCCCCUGGGCCUAUCCUAGAA-3'; miR-331-3p inhibitor, 5'-UUCUAGGAUAGGCCCAGGGGC-3'; mimic NC, 5'-UUCUCCGAACGUGUCACGUTT-3'; inhibitor NC: 5'-CAGUACUUUUGUGUAGUACAA-3'.

Dual luciferase reporter assay. The interactions between UCA1 and miR-331-3p were predicted by starBase (http://www.starbase.sysu.edu.cn). The pMirReporter plasmid (Sigma-Aldrich; Merck $\mathrm{KGaA}$ ) was used to produce the UCA1-mutated (mut) and UCA1-wild (wt). UCA1-mut or UCA1-wt, miR-NC or miR-331-3p mimics were co-transfected into U2932 cells using Lipofectamine 2000 (Invitrogen; Thermo Fisher Scientific, Inc.). After transfection at $37^{\circ} \mathrm{C}$ for $48 \mathrm{~h}$, the relative luciferase activities were detected by Dual-Luciferase reporter assay kit (Promega Corporation), according to the manufacturer's protocol. Luciferase activity was measured and normalized to Renilla luciferase activity.

MTT assay. DLBCL cells were cultured in a 96-well plate (1x10 ${ }^{4}$ cells/well) and cultured for $24,48,72$ and $96 \mathrm{~h}$. Then MTT reagent $(10 \mu \mathrm{l} ; 5 \mathrm{mg} / \mathrm{ml})$ was added to each well and incubated for another $4 \mathrm{~h}$ at $37^{\circ} \mathrm{C}$. After the supernatant was removed, dimethyl sulfoxide (DMSO) was added (100 $\mu \mathrm{l} /$ well). The absorbance value at a wavelength of $490 \mathrm{~nm}$ was measured with a microplate reader.

Transwell assays. DLBCL cell migration and invasion were assessed using Transwell chambers ( $8 \mu \mathrm{m}$; BD Biosciences). Cell invasion was detected in the upper chamber with Matrigel 
(BD Falcon; BD Biosciences). Cell migration was performed without Matrigel. First, $1 \times 10^{5}$ cells were inoculated in the upper Transwell compartment. Then, $10 \%$ FBS was added into the lower compartment. After culture at $37^{\circ} \mathrm{C}$ for $24 \mathrm{~h}$, the cells were fixed with $4 \%$ formaldehyde for $30 \mathrm{~min}$ and stained with $0.5 \%$ crystal violet for $20 \mathrm{~min}$ at room temperature. Finally, the number of migrated and invasive cells were counted under an optical microscope (magnification, x100; Olympus Corporation).

Statistical analysis. SPSS 22.0 (IBM Corp.) and GraphPad Prism 7.0 (GraphPad Software, Inc.) were used for statistical analysis. All data are presented as the mean \pm standard deviation (SD). Each experiment was performed at least three times. The paired Student's t-test was used to evaluate the differences between two groups. One-way ANOVA and Tukey's post hoc test were performed to detect the differences in multiple groups. Kaplan-Meier method and log-rank test were used to plot the survival curve of DLBCL patients. $\mathrm{P}<0.05$ was considered to indicate a statistically significant difference.

\section{Results}

UCA1 and miR-331-3p are aberrantly expressed in DLBCL. First, the expression pattern of UCA1 and miR-331-3p in DLBCL tissues was detected by RT-qPCR analysis. The results revealed that the expression of UCA1 was higher in DLBCL tissues than in normal tissues (Fig. 1A). Conversely, the expression level of miR-331-3p in DLBCL tissues was decreased compared with normal tissues (Fig. 1B). Moreover, compared with normal cell line GM12878, UCA1 was revealed to be upregulated in DLBCL cell lines (TMD8, U2932, OCI-Ly10, OCI-Ly 7 and JeKo-1), especially in U2932 cells (Fig. 1C). Hence, in the following study, U2932 cells were selected as the research representative of DLBCL cells. On the contrary, miR-331-3p expression was lower in the DLBCL cell lines than in the GM12878 cells (Fig. 1D). All these data indicated that UCA1 and miR-331-3p may participate in the progression of DLBCL.

Knockdown of UCA1 inhibits cell proliferation, migration and invasion in DLBCL. Next, the specific role of UCA1 in the progression of DLBCL was investigated. According to the average expression of UCA1, the DLBCL patients were divided into a high-UCA1 expression group $(n=21)$ and a low-UCA1 expression group $(\mathrm{n}=17)$. The survival time in both groups was investigated by Kaplan-Meier. The results revealed that DLBCL patients with high UCA1 expression had shorter survival time than the patients in the low-expression group (Fig. 2A). Moreover, it was determined that UCA1 was closely associated with IPI score (Table I). Then, si-UCA1 was transfected into U2932 cells, and it was revealed that the expression level of UCA1 was significantly decreased by si-UCA1 transfection (Fig. 2B). Subsequently, MTT and Transwell assays were used to assess DLBCL cell activity. The results revealed that cell proliferation of U2932 cells was inhibited by depletion of UCA1 (Fig. 2C). Similarly, it was observed that depletion of UCA1 decreased the number of migrated and invaded U2932 cells (Fig. 2D and E). The present findings demonstrated that UCA1 expression affected the survival time of DLBCL patients and silencing of UCA1 suppressed cell proliferation, migration and invasion of DLBCL cells.

UCA1 can act as a sponge of miR-331-3p in DLBCL. Starbase database predicted that there were complementary sequences between UCA1 and miR-331-3p (Fig. 3A). To verify this prediction, miR-331-3p mimics or miR-331-3p inhibitor was transfected into U2932 cells. RT-qPCR results revealed that the expression of miR-331-3p was significantly increased by miR-331-3p mimics, but reduced by miR-331-3p inhibitor (Fig. 3B). Then, a Dual Luciferase Reporter assay was performed to assess the relative luciferase activity of UCA1. It was observed that the luciferase activity of UCA1-wt was decreased by miR-331-3p, but there was no change in UCA1-mut (Fig. 3C). Moreover, the relationship between UCA1 and miR-331-3p was explored. The results revealed that $\mathrm{miR}-331-3 p$ was downregulated by UCA1 vector, while it was upregulated by si-UCA1 transfection (Fig. 3D). Furthermore, miR-331-3p mimics decreased the expression level of UCA1, while miR-331-3p inhibitor increased the expression level of UCA1 (Fig. 3E). Furthermore, Pearson's correlation analysis revealed that there was a negative correlation between UCA1 and miR-331-3p (Fig. 3F). These experimental results indicated that UCA1 can be a sponge to regulate miR-331-3p expression in DLBCL.

miR-331-3p overexpression inhibits cell proliferation, migration and invasion in DLBCL cells. To investigate the role of miR-331-3p on the progression of DLBCL cells, miR-331-3p mimics were transfected into U2932 cells. Functionally, it was revealed that miR-331-3p mimics inhibited the cell proliferation capability of U2932 cells (Fig. 4A). Moreover, when U2932 cells were transfected with miR-331-3p mimics, a significant decrease of migrated and invasive cells were observed in the Transwell migration and invasion assays, compared with miR-NC (Fig. 4B and C). Additionally, downregulation of miR-331-3p was revealed to be associated with LDH ratio in DLBCL patients (Table II). Collectively the data demonstrated that miR-331-3p upregulation prevented DLBCL cell progression.

UCA1 regulates $D L B C L$ cell progression by competitively binding with miR-331-3p. To explore the role of DLBCL/ miR-331-3p on the progression of DLBCL, si-UCA1 were transfected into U2932 cells with miR-331-3p inhibitor. As revealed in Fig. 5A, the expression of miR-331-3p was increased by si-UCA1, while it was reduced by miR-331-3p inhibitor with UCA1 knockdown. Next, functional experiments on the UCA1/miR-331-3p axis were conducted. It was revealed that the inhibitory effect of si-UCA1 on cell proliferation was restored by miR-331-3p inhibitor (Fig. 5B). Moreover, the suppression of cell migration ability was reversed by miR-331-3p inhibitor (Fig. 5C). Similarly, miR-331-3p inhibitor had the same effect on cell invasion ability (Fig. 5D). Consequently, the present data indicated that knockdown of UCA1 had a negative effect on DLBCL cell progression by inhibiting miR-331-3p expression.

\section{Discussion}

Studies have revealed that various lncRNAs are involved in the tumorigenesis and development of DLBCL, and 
Table I. Associations between the expression level of UCA1 and clinical characteristics of DLBCL patients $(\mathrm{n}=38)$.

\begin{tabular}{|c|c|c|c|c|}
\hline \multirow[b]{2}{*}{ Clinical characteristics } & \multirow[b]{2}{*}{ Number of cases $n=38$} & \multicolumn{2}{|c|}{ UCA1 expression } & \multirow[b]{2}{*}{ P-value } \\
\hline & & Low $(n=17)$ & $\operatorname{High}(n=21)$ & \\
\hline Age (years) & & & & 0.389 \\
\hline$>60$ & 15 & 8 & 7 & \\
\hline$\leq 60$ & 23 & 9 & 14 & \\
\hline Sex & & & & 0.973 \\
\hline Male & 18 & 8 & 10 & \\
\hline Female & 20 & 9 & 11 & \\
\hline Ann Arbor stage & & & & 0.618 \\
\hline I-II & 24 & 10 & 14 & \\
\hline III-IV & 14 & 7 & 7 & \\
\hline Clinical stage & & & & 0.899 \\
\hline I-II & 13 & 6 & 7 & \\
\hline III-IV & 25 & 11 & 14 & \\
\hline LDH ratio & & & & 0.054 \\
\hline$\leq 1$ & 20 & 6 & 14 & \\
\hline$>1$ & 18 & 11 & 7 & \\
\hline IPI score & & & & $0.021^{\mathrm{a}}$ \\
\hline $0-2$ & 26 & 8 & 18 & \\
\hline $3-5$ & 12 & 9 & 3 & \\
\hline
\end{tabular}

${ }^{\mathrm{a}} \mathrm{P}<0.05$, indicating a significant difference. UCA1, urothelial cancer associated 1; DLBCL, diffuse large B-cell lymphoma; LDH, lactate dehydrogenase; IPI, International Prognostic Index.
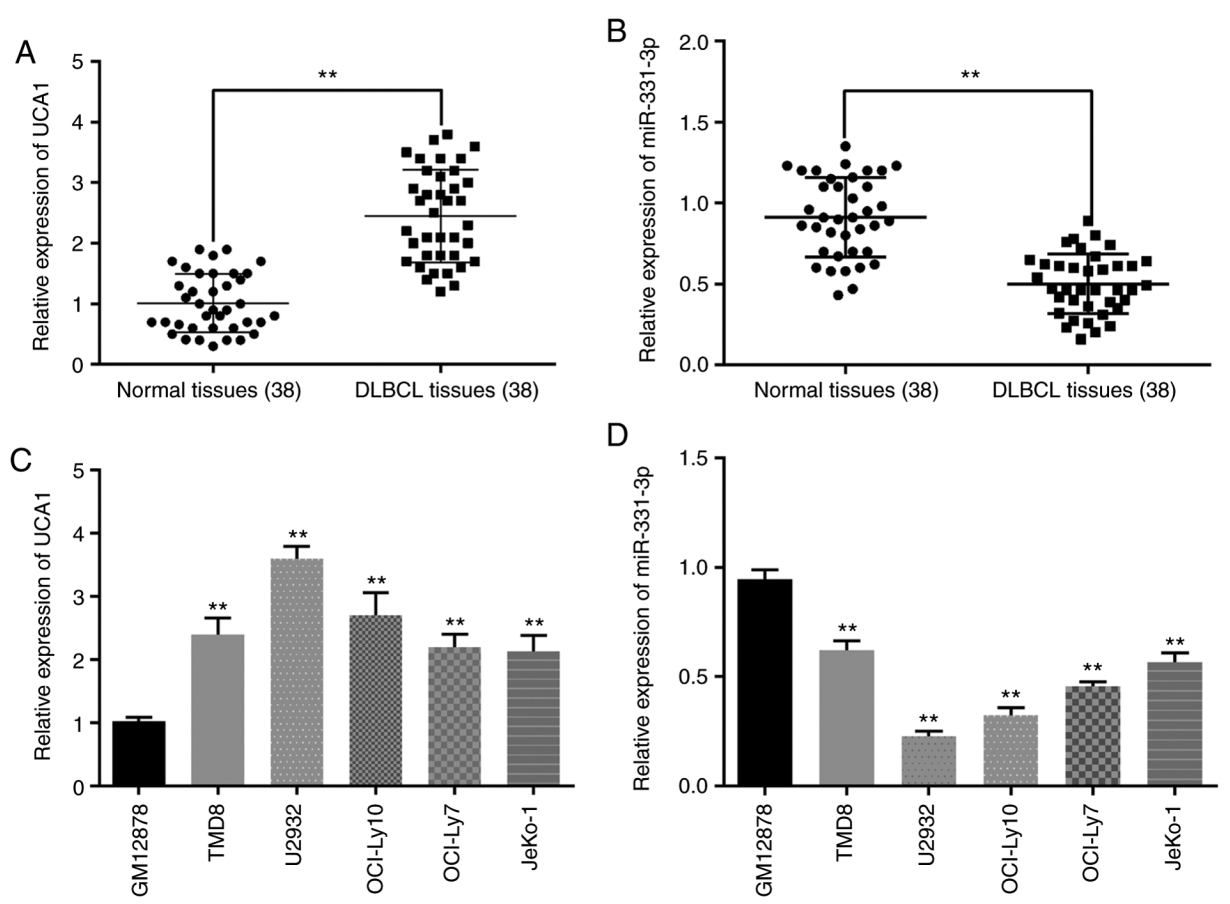

Figure 1. UCA1 and miR-331-3p are aberrantly expressed in DLBCL. (A) UCA1 was upregulated in DLBCL tissues. (B) miR-331-3p was downregulated in DLBCL tissues. (C) The expression of UCA1 was higher in DLBCL cell lines than in GM12878 cells. (D) miR-331-3p was downregulated in DLBCL cell lines. ${ }^{* *} \mathrm{P}<0.01$. UCA1, urothelial cancer associated 1; miR-331-3p, microRNA-331-3p; DLBCL, diffuse large B-cell lymphoma.

act as tumor suppressors or tumor carcinogens (20). For example, DBH-AS1 was confirmed to drive DLBCL cell progression, and could be a therapeutic target for the treatment of DLBCL (21). Conversely, NONHSAG026900 suppressed 
Table II. Associations between the expression level of miR-331-3p and clinical characteristics of DLBCL patients ( $\mathrm{n}=38$ ).

miR-331-3p expression

Clinical characteristics

Number of cases $n=38$

Low $(\mathrm{n}=18)$

High $(\mathrm{n}=20)$

P-value

Age (years)

$>60$

15

23

7

0.944

$\leq 60$

11

8

Sex

18

8

12

Male

20

10

0.732

Female

I-II

Clinical stage

I-II

III-IV

25

LDH ratio

$$
\leq 1
$$

$>1$

20

18

IPI score

0-2

3-5
26

12
4

14

13

5

15

3
10

10

12

8

9

11

$0.022^{\mathrm{a}}$

7

13

0.061

${ }^{a} \mathrm{P}<0.05$, indicating a significant difference. miR-331-3p, microRNA-331-3p; DLBCL, diffuse large B-cell lymphoma; LDH, lactate dehydrogenase; IPI, International Prognostic Index.
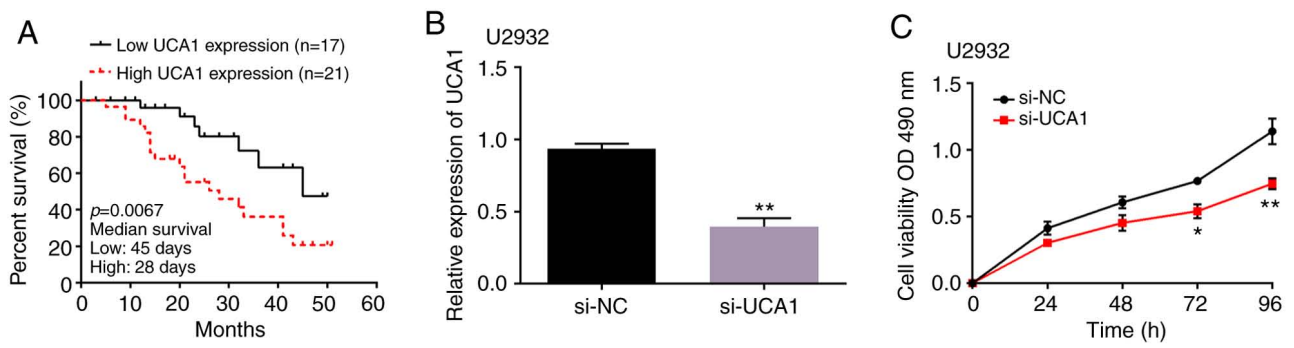

D
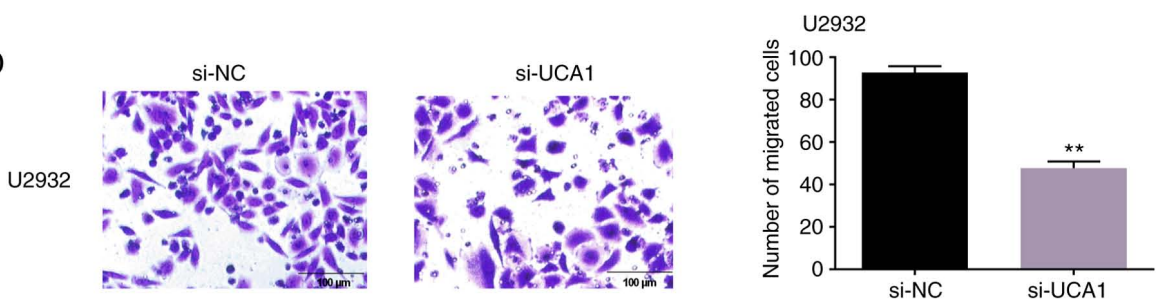

E si-NC si-UCA1
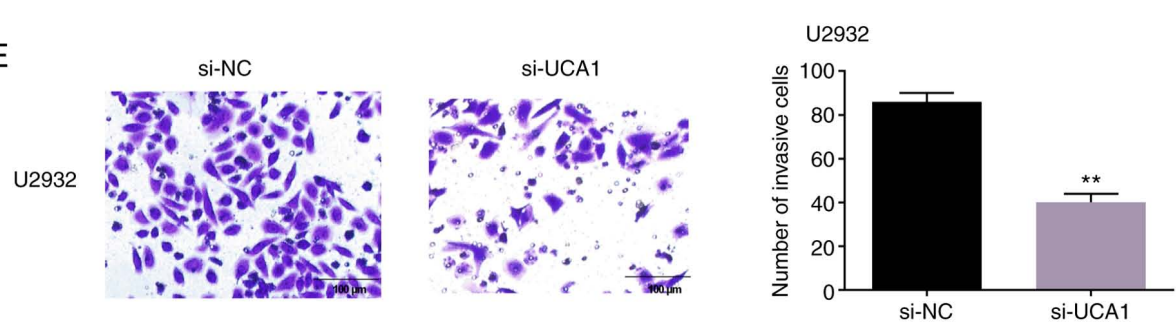

Figure 2. Knockdown of UCA1 inhibits cell proliferation, migration and invasion of DLBCL. (A) The survival time of patients in the UCA1 high-expression group was shorter than that in the UCA1 low-expression group. (B) The expression of UCA1 was decreased by silencing of UCA1. (C) Cell proliferation of U2932 cells was suppressed by si-UCA1 (scale bar, $100 \mu \mathrm{m}$ ). (D) Cell migration of U2932 cells was suppressed by si-UCA1 (scale bar, $100 \mu$ m). (E) Cell invasion of U2932 cells was inhibited by si-UCA1. ${ }^{*} \mathrm{P}<0.05$ and ${ }^{* *} \mathrm{P}<0.01$ compared to si-NC. UCA1, urothelial cancer associated 1 ; DLBCL, diffuse large B-cell lymphoma; si-, small interfering; NC, negative control; OD, optical density. 

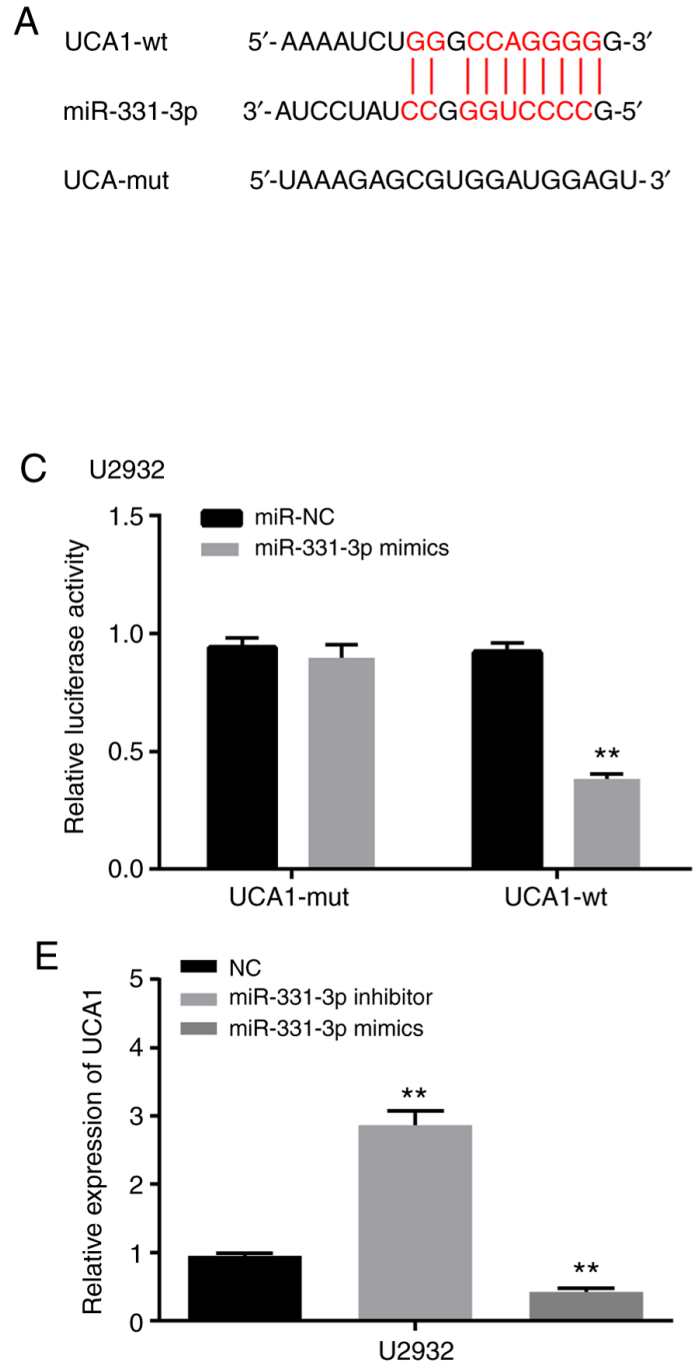
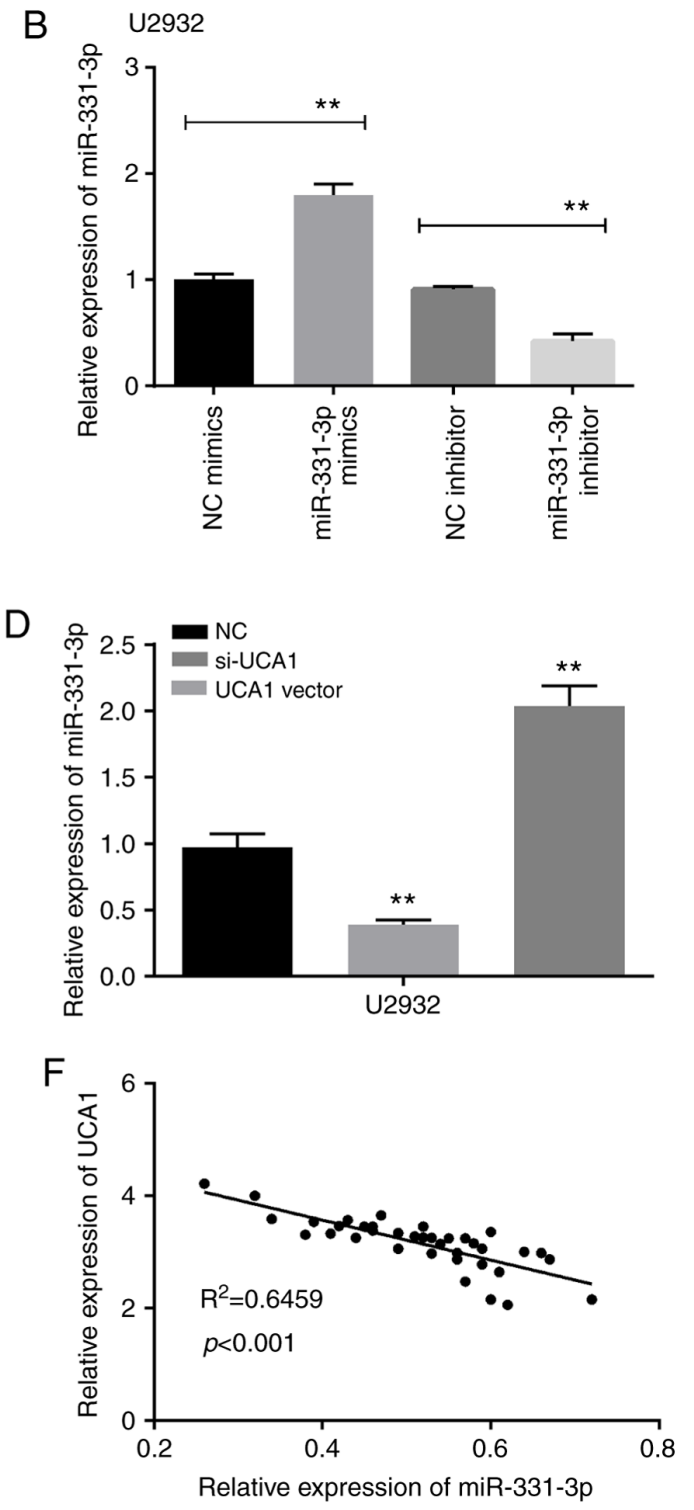

Figure 3. UCA1 can act as a sponge of miR-331-3p in DLBCL. (A) Binding sites between UCA1 and miR-331-3p. (B) The expression of miR-331-3p was increased by miR-331-3p mimics, while it was reduced by miR-331-3p inhibitor. (C) The luciferase activity of UCA1-wt was significantly reduced by miR-331-3p mimics, while there was no change on UCA1-mut. (D) The expression of miR-331-3p was increased by si-UCA1, but reduced by UCA1 vector. (E) The expression of UCA1 was increased by miR-331-3p inhibitor, while it was decreased by miR-331-3p mimics. (F) There was a negative correlation between UCA1 and miR-331-3p. ${ }^{* *} \mathrm{P}<0.01$. UCA1, urothelial cancer associated 1; miR-331-3p, microRNA-331-3p; DLBCL, diffuse large B-cell lymphoma; wt, wild-type; mut, mutated; NC, negative control; si-, small interfering.

DLBCL cell proliferation and cell activity, and could be a favorable biomarker in DLBCL. LncUCA1 has been revealed to be aberrantly expressed in several human tumors, and to participate in tumor tumorigenesis and development. However, the role of UCA1 remains unclear. In the present research, it was revealed that the expression of UCA1 was significantly upregulated in DLBCL tissues and cell lines. Consistent with our results, UCA1 was also upregulated in gastric (22) and adrenocortical cancer (23). In glioma, UCA1 was detected to drive cell proliferation, migration and EMT (24). In agreement with previous studies, functional experiments revealed that silencing of UCA1 suppressed U2932 cell proliferation, and increased the number of migrated and invaded cells. Similarly, UCA1 was demonstrated to be actively involved in tumor initiation and progression, and act as an oncogene in multiple myeloma (25) and prostate cancer (26). The present results indicated that UCA1 knockdown inhibited cell proliferation, migration and invasion in DLBCL.

miRNAs are a type of endogenous non-coding regulatory RNAs with a length of 20-25 nucleotides. Researches have revealed that miRNAs regulate gene expression through sequence-specific translation or mRNA cleavage, and are involved in a series of important biological processes such as cell proliferation, differentiation, and apoptosis $(27,28)$. In recent years, research on the relationship between DLBCL and miRNAs has made great advances. For example, Ting et al (29) systematically analyzed a large number of studies and found that miR-155, miR-334, miR-21, miR-146b-5p and miR-17/92 clusters have certain value in predicting the chemotherapy response in DLBCL. In previous research, UCA1 was revealed to competitively bind with several miRNAs $(13,30)$. Gao et al (31) confirmed that UCA1 facilitated thyroid cancer 

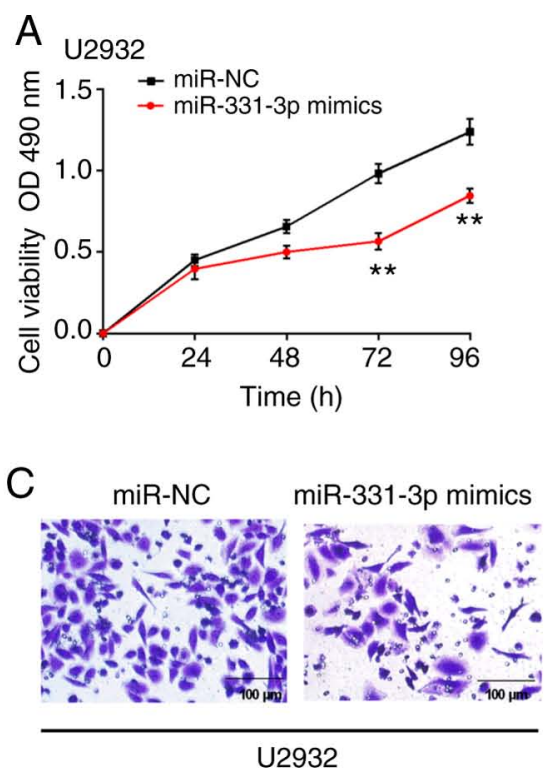

B
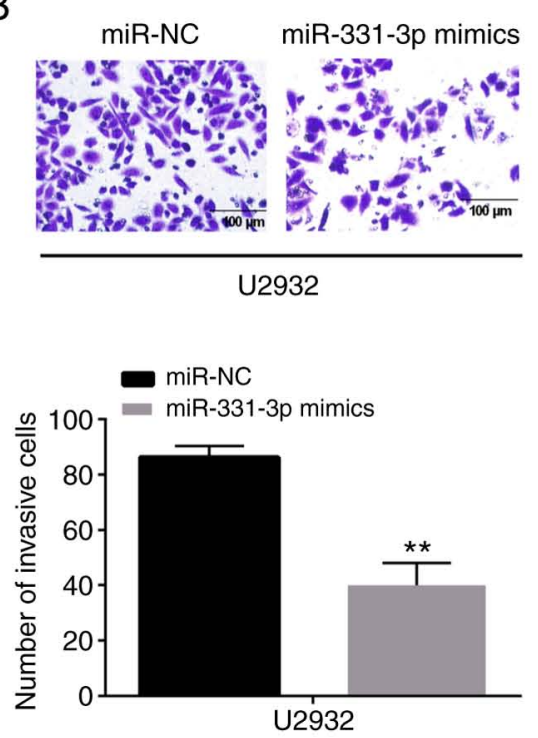

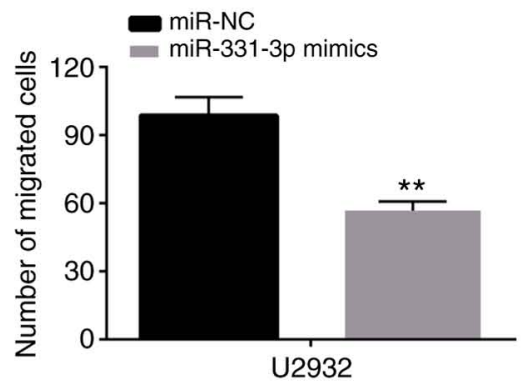

Figure 4. miR-331-3p prevents cell proliferation, migration and invasion of DLBCL. (A) Cell proliferation of U2932 cells was inhibited by miR-331-3p mimics. (B and C) miR-331-3p mimics suppressed cell migration and invasion of U2932 cells (scale bar, $100 \mu \mathrm{m}$ ). ${ }^{* *} \mathrm{P}<0.01$. miR-331-3p, microRNA-331-3p; DLBCL, diffuse large B-cell lymphoma; NC, negative control; OD, optical density.

A ले

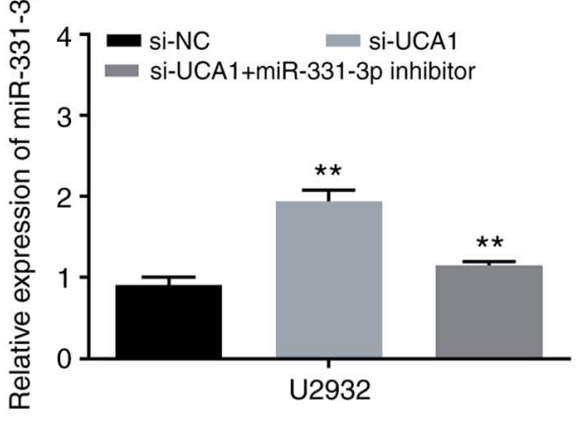

C si-NC
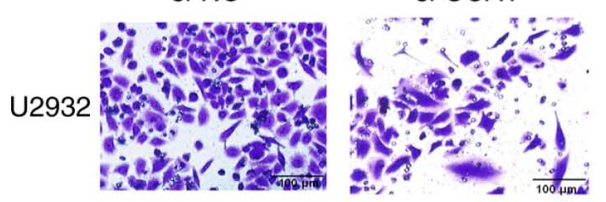

si-UCA1+ miR-331-3p inhibitor

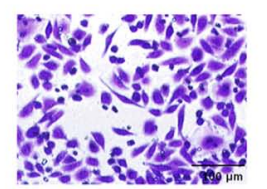

Si-UCA1+

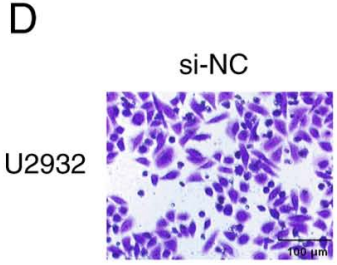

Si-UCA1

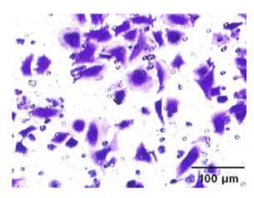

B
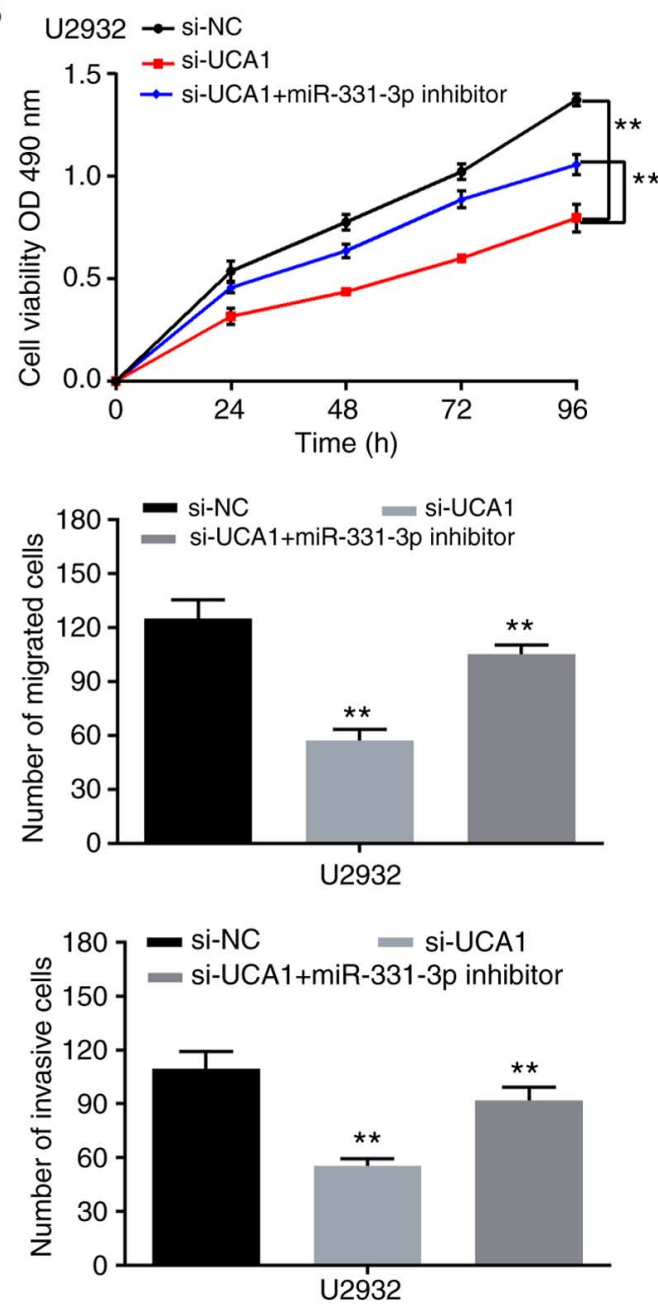

Figure 5. UCA1 regulates DLBCL cell progression by competitively binding with miR-331-3p. (A) The expression of miR-331-3p in U2932 cells transfected with si-UCA1 and miR-331-3p inhibitor. (B) Cell proliferation was suppressed by si-UCA1, while it was increased by miR-331-3p inhibitor. (C and D) Cell migration and invasion were inhibited by si-UCA1 transfection, but reversed by miR-331-3p inhibitor (scale bar, $100 \mu \mathrm{m}$ ). "* P<0.01. UCA1, urothelial cancer associated 1; DLBCL, diffuse large B-cell lymphoma; miR-331-3p, microRNA-331-3p; si-, small interfering; NC, negative control; OD, optical density. 
cell ability and metastasis by decreasing the expression of miR-497-3p. In addition, UCA1 was revealed to directly target miR-495 $(32,33)$ and miR-185-5p (34). In the present study, UCA1 was revealed to act as a molecular sponge of miR-331-3p, which was consistent with previous findings in multiple myeloma (35). In addition, there was a negative relationship between UCA1 and miR-331-3p in DLBCL. Moreover, the expression level and role of miR-331-3p in DLBCL were detected. The results revealed that miR-331-3p expression was decreased in DLBCL tissues and cell lines. Furthermore, functional experiments revealed that overexpression of miR-331-3p inhibited U2932 cell proliferation, migration and invasion. In addition, miR-331-3p inhibitor was revealed to reverse the inhibitory effect on cell proliferation and metastasis which was stimulated by si-UCA1. According to the present findings, UCA1 had a negative effect on DLBCL cell progression by inhibiting miR-331-3p expression.

In conclusion, upregulation of UCA1 and downregulation of miR-331-3p were revealed in DLBCL tissues and cell lines. Moreover, the present findings firstly indicated that knockdown of UCA1 suppressed cell proliferation, migration and invasion by competitively binding with miR-331-3p in DLBCL. Combined with all findings, it is suggested that UCA1 could be a target for treatment and prognosis of DLBCL.

\section{Acknowledgements}

Not applicable.

\section{Funding}

The present study was supported by the Development Plan of Medical and Health Science and Technology of Shandong province (grant no. 2016WS0472) and the Key R\&D Program Projects of Shandong Province (grant no. 2018GSF118203).

\section{Availability of data and materials}

The datasets used during the present study are available from the corresponding author upon reasonable request.

\section{Authors' contributions}

MZ, YD, JS and HC designed and conceived the experiments. MZ, DZ and XD performed most of the experiments. MZ collected and analyzed the clinical and pathological data. All authors read and approved the manuscript.

\section{Ethics approval and consent to participate}

The present study was approved by the Institutional Human Experimentation and Ethics Committee of Chengwu People's Hospital (Heze, China). Moreover, the guidelines of the declaration of Helsinki were strictly adhered to during the study. Prior to the start of the study, all patients signed written informed consent.

\section{Patient consent for publication}

Not applicable.

\section{Competing interests}

The authors declare that they have no competing interests.

\section{References}

1. García-Morales E, Lázaro-Martínez JL, Martínez-Hernández D, Aragón-Sánchez J, Beneit-Montesinos JV and González-Jurado MA: Impact of diabetic foot related complications on the Health Related Quality of Life (HRQol) of patients-a regional study in Spain. Int J Low Extrem Wounds 10: 6-11, 2011

2. Patterson E: The spending power after NFIB: New direction, or medicaid exception? SMU Law Rev 68: 385-426, 2015.

3. Miao Y, Medeiros LJ, Xu-Monette ZY, Li J and Young KH: Dysregulation of cell survival in diffuse large B cell lymphoma: Mechanisms and therapeutic targets. Front Oncol 9: 107, 2019.

4. Huarte M: The emerging role of lncRNAs in cancer. Nat Med 21: 1253-1261, 2015.

5. Gil N and Ulitsky I: Regulation of gene expression by cis-acting long non-coding RNAs. Nat Rev Genet 21: 102-117, 2020.

6. Liu J, Xu R, Mai SJ, Ma YS, Zhang MY, Cao PS, Weng NQ, Wang RQ, Cao D, Wei W, et al: LncRNA CSMD1-1 promotes the progression of hepatocellular carcinoma by activating MYC signaling. Theranostics 10: 7527-7544, 2020.

7. Huang D, Chen J, Yang L, Ouyang Q, Li J, Lao L, Zhao J, Liu J, $\mathrm{Lu}$ Y, Xing Y, et al: NKILA IncRNA promotes tumor immune evasion by sensitizing T cells to activation-induced cell death. Nat Immunol 19: 1112-1125, 2018.

8. Mehrpour Layeghi S, Arabpour M, Esmaeili R, Naghizadeh MM Tavakkoly Bazzaz J and Shakoori A: Evaluation of the potential role of long non-coding RNA LINC00961 in luminal breast cancer: A case-control and systems biology study. Cancer Cell Int 20: 478, 2020.

9. Goie TT and Naidoo M: Awareness of diabetic foot disease amongst patients with type 2 diabetes mellitus attending the chronic outpatients department at a regional hospital in Durban, South Africa. Afr J Prim Health Care Fam Med 8: e1-e8, 2016.

10. Zhao L, Liu Y, Zhang J, Liu Y and Qi Q: LncRNA SNHG14/miR5590-3p/ZEB1 positive feedback loop promoted diffuse large B cell lymphoma progression and immune evasion through regulating PD-1/PD-L1 checkpoint. Cell Death Dis, 10: 731, 2019.

11. Pichu S, Patel BM, Apparsundaram S and Goyal RK: Role of biomarkers in predicting diabetes complications with special reference to diabetic foot ulcers. Biomark Med 11: 377-388, 2017.

12. Chalatsa I, Arvanitis DA, Koulakiotis NS, Giagini A, Skaltsounis AL, Papadopoulou-Daifoti Z, Tsarbopoulos A and Sanoudou D: The Crocus sativus compounds trans-crocin 4 and trans-crocetin modulate the amyloidogenic pathway and tau misprocessing in Alzheimer disease neuronal cell culture models. Front Neurosci 13: 249, 2019.

13. Hu M and Yang J: Down-regulation of lncRNA UCA1 enhances radiosensitivity in prostate cancer by suppressing EIF4G1 expression via sponging miR-331-3p. Cancer Cell Int 20: 449, 2020.

14. Kocaman G, Altinoz E, Erdemli ME, Gul M, Erdemli Z, Gul S and Bag HG: Protective effects of crocin on biochemistry and histopathology of experimental periodontitis in rats. Biotech Histochem 94: 366-373, 2019.

15. Zhu A, Lao C, Wang Z, Chen Y and Bai C: Characterization of crocetin-monoglucuronide as a neuron-protective metabolite of crocin-1. Mol Nutr Food Res: Apr 29, 2019 (Epub ahead of print).

16. Khalatbari-Mohseni A, Banafshe HR, Mirhosseini N, Asemi Z, Ghaderi A and Omidi A: The effects of crocin on psychological parameters in patients under methadone maintenance treatment: A randomized clinical trial. Subst Abuse Treat Prev Policy 14: 9, 2019.

17. Deng L, Li J, Lu S and Su Y: Crocin inhibits proliferation and induces apoptosis through suppressing MYCN expression in retinoblastoma. J Biochem Mol Toxicol 33: e22292, 2019.

18. Colapietro A, Mancini A, D'Alessandro AM and Festuccia C: Crocetin and crocin from saffron in cancer chemotherapy and chemoprevention. Anticancer Agents Med Chem 19: 38-47, 2019.

19. Livak KJ and Schmittgen TD: Analysis of relative gene expression data using real-time quantitative PCR and the 2(-Delta Delta C(T)) method. Methods 25: 402-408, 2001.

20. Huang X, Qian W and Ye X: Long noncoding RNAs in diffuse large B-cell lymphoma: Current advances and perspectives Onco Targets Ther 13: 4295-4303, 2020. 
21. Demurtas OC, Frusciante S, Ferrante P, Diretto G, Azad NH, Pietrella M, Aprea G, Taddei AR, Romano E, Mi J, et al: Candidate enzymes for saffron crocin biosynthesis are localized in multiple cellular compartments. Plant Physiol 177: 990-1006, 2018.

22. Taherkhani T, Asghari Zakaria R, Omidi M and Zare N: Effect of ultrasonic waves on crocin and safranal content and expression of their controlling genes in suspension culture of saffron (Crocus sativus L). Nat Prod Res 33: 486-493, 2019.

23. Altinoz E, Erdemli ME, Gul M, Aksungur Z, Gul S, Bag HG Kaya GB and Turkoz Y: Neuroprotection against $\mathrm{CCl} 4$ induced brain damage with crocin in Wistar rats. Biotech Histochem 93: 623-631, 2018

24. Yorgun MA: Effects of crocin on diabetic maculopathy: A placebo-controlled randomized clinical trial. Am J Ophthalmol 204: 141-142, 2019.

25. Roshankhah S, Jalili C and Salahshoor MR: Effects of crocin on sperm parameters and seminiferous tubules in diabetic rats. Adv Biomed Res 8: 4, 2019.

26. Mozaffari S, Ramezany Yasuj S, Motaghinejad M, Motevalian M and Kheiri R: Crocin acting as a neuroprotective agent against methamphetamine-induced neurodegeneration via CREB-BDNF signaling pathway. Iran J Pharm Res 18: 745-758, 2019.

27. Komoll RM, Hu Q, Olarewaju O, von Döhlen L, Yuan Q, Xie Y, Tsay HC, Daon J, Qin R, Manns MP, et al: MicroRNA-342-3p is a potent tumour suppressor in hepatocellular carcinoma. J Hepatol: Jul 30, 2020 (Online ahead of print).

28. Fuertes T, Ramiro AR and de Yebenes VG: miRNA-based therapies in B cell non-hodgkin lymphoma. Trends Immunol 41 932-947, 2020

29. Ting CY, Liew SM, Price A, Gan GG, Bee-Lan Ong D, Tan SY and Bee PC: Clinical significance of aberrant microRNAs expression in predicting disease relapse/refractoriness to treatment in diffuse large B-cell lymphoma: A meta-analysis. Critical Reviews in Oncology/Hematology, 144: 102818, 2019.
30. Guo Z, Wang X, Yang Y, Chen W, Zhang K, Teng B, Huang C, Zhao $Q$ and Qiu Z: Hypoxic tumor-derived exosomal long noncoding RNA UCA1 promotes angiogenesis via miR-96-5p/AMOTL2 in pancreatic cancer. Mol Ther Nucleic Acids: Aug 25, 2020 (Epub ahead of print).

31. Gao H, Yang JY, Tong LX, Jin $\mathrm{H}$ and Liu CZ: Long noncoding RNA UCA1 promotes proliferation and metastasis of thyroid cancer cells by sponging miR-497-3p. Eur Rev Med Pharmacol Sci, 24: 7555, 2020.

32. Mohammadzadeh L, Hosseinzadeh H, Abnous K and Razavi BM: Neuroprotective potential of crocin against malathion-induced motor deficit and neurochemical alterations in rats. Environ Sci Pollut Res Int 25: 4904-4914, 2018.

33. Lin L, Liu G and Yang L: Crocin improves cognitive behavior in rats with Alzheimer's disease by regulating endoplasmic reticulum stress and apoptosis. Biomed Res Int 2019: 9454913, 2019.

34. Sebastin Santhosh M, Hemshekhar M, Thushara RM, Devaraja S, Kemparaju K and Girish KS: Vipera russelli venom-induced oxidative stress and hematological alterations: Amelioration by crocin a dietary colorant. Cell Biochem Funct 31: 41-50, 2013.

35. Asanad K: Radiographic evolution of a simple renal cyst to clear cell renal cell carcinoma in three years. Urol Case Rep 32: 101212, 2020.

(i) $\odot$ This work is licensed under a Creative Commons Attribution-NonCommercial-NoDerivatives 4.0 International (CC BY-NC-ND 4.0) License. 